\title{
Symmetric Motions for Bimanual Rehabilitation
}

\author{
Hernando Gonzalez Malabet, Rafael Alvarez Robles, and Kyle B. Reed
}

\begin{abstract}
Many daily tasks require that a person use both hands simultaneously, such as moving a large book or opening the lid on a jar. Such bimanual tasks are difficult for people who have a stroke, but the tight neural coupling across the body has been hypothesized to allow individuals to self-rehabilitate by physically coupling their hands. To examine potential methods for robot-assisted bimanual rehabilitation, we performed a haptic tracking task where individuals experience a one degree of freedom trajectory on one hand and attempt to recreate it with their other hand. Despite the biomechanical and neurological symmetries present across the human body, subjects performed this task worse when working in the joint space (i.e., mirrored motion) than they did in the visually centered space. We also examined multiple input paths and show alternative rhythmic motions that may aid in rehabilitation.
\end{abstract}

\section{INTRODUCTION}

Humans have an excellent ability to synchronize the motions between both sides of their bodies. Note how easy it is to simultaneously draw a circle with each hand and how difficult it is to simultaneously draw a circle with one hand and a square with the other. These symmetric motions suggest an excellent method to allow individuals with a stroke to self-rehabilitate. A stroke in the brain typically causes motor impairments on the side of the body opposite the brain lesion while the other side retains most of its motor function. The idea of bimanual rehabilitation is to physically couple the individual's two limbs such that the healthy limb can guide the impaired limb. Such a method has been shown to provide positive results similar to other state-of-the-art rehabilitation methods [1]. One of the advantages of using both limbs is that the assistance force would come from the individual and minimal external assistance would be required from a human or robotic caregiver, which would make this an ideal solution for low cost, home-based, self-rehabilitation. Our ultimate goal in this work is to identify the bilateral interactions necessary for a robot to facilitate the physical coupling between the two arms for bimanual rehabilitation.

In this paper, we first describe some of the common methods for rehabilitation and describe why we think bimanual methods are well suited for home-based upperextremity rehabilitation. Then, in Section IV, we report on our experiments examining additional modes of bimanual interactions that we anticipate will eventually lead to homebased rehabilitation.

H. Gonzalez Malabet, R. Alvarez Robles, and K. B. Reed are with the Department of Mechanical Engineering, University of South Florida, 4202 E. Fowler Ave, Tampa, FL, 33612 \{ralvarez, hgonzale\}@mail.usf.edu, kylereed@usf.edu

This work was supported by the University of South Florida.

\section{BACKGROUND}

The goal of upper limb rehabilitation after a stroke is to allow a person to use both hands in activities of daily living. Many of the current methods found in the rehabilitation literature show positive results, but there is no clear indication that any of these methods can produce benefits greater than is possible with traditional techniques [2][3]. A common thread among all these studies is that the amount of time spent training the affected arm plays an important role in improving the functional ability of the affected arm [4]. The old saying "practice makes perfect", although cliché, is adequate in describing a good method of rehabilitation. Increased stroke training leads to improved motor relearning.

\section{A. Non-Robotic Rehabilitation}

Conventional therapies, such as the Bobath method [5] and the proprioceptive neuromuscular facilitation technique [6], have been commonly used for stroke rehabilitation. However, the evidence of brain reorganization after these therapies is limited. Constraint-induced movement therapy has been used for nearly 100 years [7]. This method is sometimes referred to as forced non-use or forced use of the paretic limb since the idea is that the healthy limb is bound and prevented from being used. This forces the individual to only use the paretic limb, which aids in cortical re-mapping of neurons from damaged brain cells to functional brain cells [8]. One advantage of forced use is that the learning occurs directly during the tasks that are to be learned; many of the methods discussed below retrain at a functional (i.e., semi-arbitrary task) level. However, forced use is unable to provide assistance, so it only works on mildly to moderately impaired individuals.

\section{B. Home-Use Rehabilitation}

The ability to train at home means patients can train more often, which may lead to faster progress and better results in motor relearning [9]. The literature has shown that guided home rehabilitation maintains patients' ability to perform activities of daily living and may lead to functional improvement [10][11]. However, Tyson and Turner [12] reported that patients are dissatisfied with their options for further training after they are discharged from rehabilitation. This result indicates that appropriate community or homebased therapy methods need to be further developed to improve functional ability after discharge.

Many of the current home-based methods use a home computer with limited accessories that cannot provide assistance forces and can only operate over a small workspace [13][14]. These methods are able to provide some benefit, but the 
rehabilitation effect is limited to people who have relatively high motor function. Many people with a stroke are unable to use these methods. Thus, we believe there is a need for additional home-based rehabilitation trainers to reach a greater population of people following a stroke. Since many of the rehabilitation methods that provide assistance show similar improvement, it would appear that there is a need for a low cost method of providing these rehabilitative motions while allowing individuals access to convenient and plentiful training. We believe rehabilitation incorporating multiple bimanual coupling modes will provide a practical method capable of increasing the length of time individuals with a stroke can train at home.

\section{Robotic Rehabilitation}

A large literature of research detailing robotic methods for upper limb rehabilitation has come about in the past fifteen years. A significant amount of the robotic rehabilitation methods focus on rehabilitating the impaired limb in planar tasks separate from the healthy hand. For the most part, these methods can be divided into two basic types: assistive forces and resistive forces [15]. Assistive forces help guide the individual toward a goal, such as a target. The assistance can either guide the individual through a path or attempt to infer their intention and assist with that motion. Assistive methods tend to generate a passive response from the individual since the robot is applying all the necessary force required to reach the target [16]. On the other hand, resistive force rehabilitation methods employ a variety of forces that require the individual to adapt, such as velocity dependent force fields [17]. The underlying idea for resistive methods is to generate an adaptation that leads to an after-effect with the correct motion.

One of the benefits of robotic devices comes from the fact that the individuals can use them for longer and more frequent periods of time in the clinic. The literature has shown that the amount of training is one of the most important factors for functional recovery after a stroke [18][19]. However, these highly actuated robotic devices may not be the ideal candidate for home use since they tend to be expensive and the large actuators are potentially dangerous. We believe bimanual rehabilitation is a good candidate for home-based self-rehabilitation.

\section{BIMANUAL REHABILITATION}

Neither a physical therapist nor a robot are, or probably ever will be, able to determine the exact path a person wants an arm to move as well as the person can. So, the idea behind bimanual rehabilitation is that the individual assists their own impaired arm with their healthy arm through a physical coupling with robotic assistance. During arm movements, the same neural signal is sent from the brain to the arms and, since the arms are constrained to move together, the proprioceptive feedback will be similar between the two sides of the brain [20][21]. The motion and neural symmetries should reinforce the motor pathways used to generate this motion.

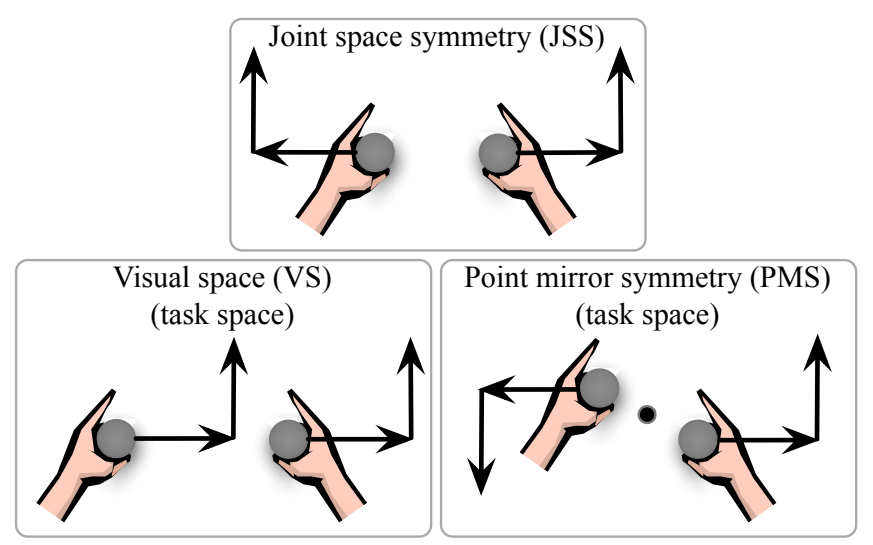

Fig. 1. Three examples of symmetry modes that can be used in bimanual rehabilitation: joint space symmetry (JSS) where the motions are mirrored and the joints on each limb follow the same angles, visual symmetry (VS) where the hands move in the same Cartesian directions, and point mirror symmetry (PMS) where the hands rotate around an arbitrary point in space.

The bimanual symmetric motions can exist in multiple reference frames; three of these symmetry types are shown in Fig. 1. The most common bimanual training mode for rehabilitation is mirror or joint space symmetry (JSS) since the joints on each side of the body are the same during movements. The brain can duplicate the efferent commands to control the joints on both limbs. Several devices have successfully implemented this mode to show rehabilitative benefits. The Mirror Image Movement Enabler [20] measures the position of the healthy arm and mirrors the motion to the impaired arm using a PUMA 560. The BiManuTrack [22] also works to mirror the movements of the two limbs, but for forearm and wrist movements and has similarly shown positive results. The BATRAC [23] is an unassisted bimanual arm trainer where individuals move their hands on two independent tracks with in-phase and out-of-phase pushing/pulling rhythmic motions. The individuals are instructed to move their arms at a comfortable frequency.

Another type of symmetry is point mirror symmetry (PMS), where the hands move simultaneously around an arbitrary point in space, much like turning a steering wheel. Johnson et al. [24] developed a driving task designed to use both arms to prevent the adverse effects of learned non-use of the impaired arm. Their method encouraged the subjects to generate coordinated beneficial motions with both arms. Without the training, subjects would typically generate counter-productive forces, which would require the healthy arm to produce a larger force.

An additional symmetry mode is visual symmetry (VS) where the hands move in similar visual paths [25], such as moving an object from one location to another [26]. The Reha-Slide [27] allows rehabilitation using visual symmetry using a sliding bar to connect the two hands.

Many activities of daily living require combinations of symmetric bimanual movements, but most rehabilitation devices tend to focus on one type. For adequate training for real world tasks, individuals with a stroke should practice each symmetry mode to prepare for different tasks [15]. All 
planar bimanual motions can be completed by combining VS with PMS, but JSS cannot be combined with either of the other two individually to provide all motions in a plane. From a task-centered standpoint, JSS is not the most obvious symmetry mode, but it has biomechanical and neurological symmetries that make it beneficial for training.

Although bimanual rehabilitation has been successfully implemented in clinical devices, there are several fundamental unanswered questions that could aid in the development of a bimanual home-based self-rehabilitation device for people with a stroke. One fundamental question is the type of symmetry to be employed. Daily bimanual tasks are often completed in visual reference frames: turning a steering wheel or opening the lid of a jar requires symmetric motions about an arbitrary point in space (PMS); moving a large object requires both hands to move through space at the same time (VS). It is currently unclear which mode or modes are most appropriate for upper limb rehabilitation following a stroke.

Another open question is the speed and complexity of the path. Previous studies have used rhythmic bimanual motions for rehabilitation [23][1], but the users were able to choose their own preferred speed. The authors are not aware of any previous study on the effect of different desired trajectories. The following experiment seeks to determine which types of paths and which symmetry modes are potentially most suitable for rehabilitation by first examining how healthy subjects are able to complete these tasks. Once healthy individuals are better understood, we will test these methods on individuals with a stroke.

\section{HAPTIC TRACKING}

To examine the differences between completing a bimanual task of varying degree of difficulty in two symmetry modes, five healthy individuals completed a physical path tracking task. The fundamental method of this experiment was to compare how well an individual could recreate a one degree of freedom path in one arm while a robot guided the other arm through an input trajectory. One of the reference frames tested is visual symmetry (VS) where the hands always move the same direction through the visual space. The second frame is joint space symmetry (JSS) where the joints on each limb follow the same angles. We chose these two symmetry modes since the results would be directly comparable along one straight line.

\section{A. Procedure}

Subjects sat down in front of two Phantom Omni force feedback devices and held onto one Omni with each hand. The Omni interacting with their left hand applied a force guiding the individual through a trajectory. The trajectory was a single or double superimposed sine wave in the lateral direction. Note that the anterior/posterior and vertical motions would be the same between VS and JSS. The guiding force was applied based on the difference between the desired path position and the actual hand position: $F=k\left(x_{\text {desired }}-x_{\text {measured }}\right)$, where $k$ is $200 \mathrm{~N} / \mathrm{m}$. The result of the virtual spring force provides a firm, but not overpowering, force that individuals can follow. The second Omni on the subject's right hand did not provide any force; subjects were instructed to recreate the path applied to the left hand with their right hand.

We tested five subjects: two females and three males, ages $20-25$, and all right handed. None of the individuals tested have any impairment that would limit their motion.

The difficulty of the task varied based on eight input trajectories: three were single frequencies, $0.5 \mathrm{~Hz}, 1.0 \mathrm{~Hz}$, and $2.0 \mathrm{~Hz}$, two were superimposed harmonic frequencies, $0.5 \& 1.0 \mathrm{~Hz}$ and $0.5 \& 1.5 \mathrm{~Hz}$, and three were superimposed non-harmonic (pseudo random) frequencies, $0.7 \& 1.1 \mathrm{~Hz}$, $1.1 \& 1.7 \mathrm{~Hz}$, and $1.7 \& 2.1 \mathrm{~Hz}$. These frequency combinations were chosen to represent a range of difficulties that could be used for a rehabilitation task. Although there have been some bimanual rehabilitation studies showing the benefits of using rhythmic bimanual motions [23][1], these studies used the user's single preferred speed.

Each of the eight frequencies were performed in the following four modes: JSS with eyes open, VS with eyes open, JSS with eyes closed, and VS with eyes closed. The experiment was performed with eyes open and eyes closed to assess whether the individuals were using proprioception or visual perception. The trial type and the order of the frequencies were applied in a different random order to each subject. The trial type and the order of the frequencies were applied in a different random order to each subject. Our analysis did not show any statistically significant learning effects over the fifteen minute experiment. Each trial was a unique combination of the symmetry type, input frequency, and eye state. Each unique trial was tested once.

The trajectory was applied for 23 seconds with a two second linear ramp up period. We performed a Fast Fourier Transform to analyze the resulting motion. Each of the 32 individual trials was evaluated based on how successfully the subjects were able to follow the trajectory. Each trial was categorized into one of the five following categories (examples shown in Fig. 2):

1) Subject successfully followed: The subject repeated the trajectory precisely and did not show any irregularities.

2) Subject successfully followed after an initial delay: The subject spent approximately 4 seconds attempting to match the trajectory. During the remaining time, the subject followed precisely.

3) Subject followed the pattern with considerable errors: The subject consistently lagged behind the input trajectory or the subject spent a considerable amount of time not following the trajectory.

4) Subject followed the frequency, but created other frequencies: The subject followed the trajectory in some parts, but created other frequencies; this typically happened because the subject made many corrective movements trying to catch up to the trajectory.

5) Subject was not able to follow the frequency: The subject was unable to either determine or replicate the trajectory and the motions did not match the trajectory. 

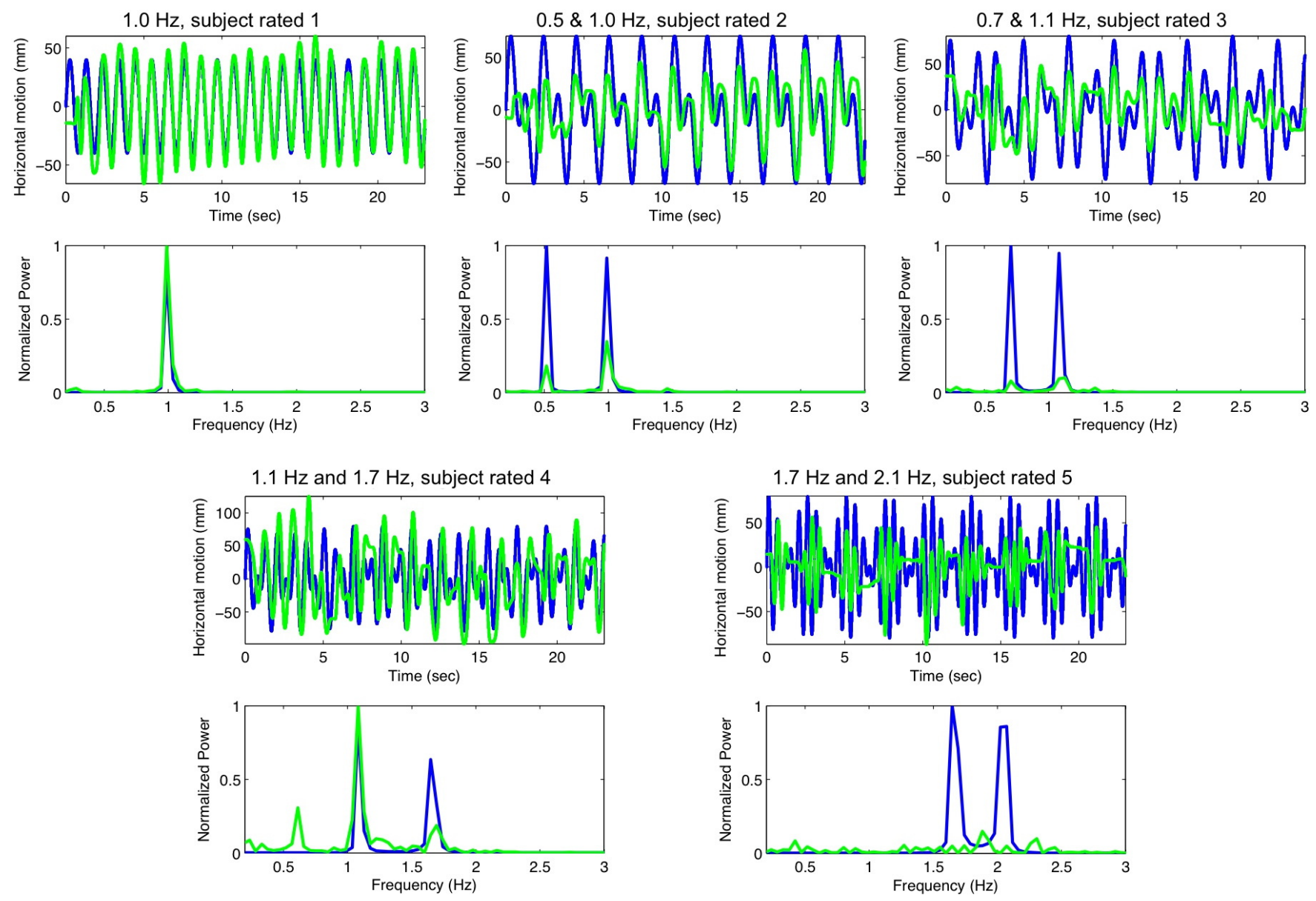

Fig. 2. Five example trajectories of subjects recreating the motion applied to one hand with the other hand shown on top. The bottom figures show a Fast Fourier Transform of the input and subjects' recreated motions. Blue (dark) lines represent the input trajectory applied to one hand and green (light) lines represent the recreated motions from the subject's other hand.

TABLE I

RESULTS FOR THE HAPTIC TRACKING TASK (LOWER NUMBER INDICATES BETTER PERFORMANCE)

\begin{tabular}{|l|c|c|c|c|c|c|c|c||c|}
\hline Sym., eyes & $0.5 \mathrm{~Hz}$ & $1.0 \mathrm{~Hz}$ & $2.0 \mathrm{~Hz}$ & $0.5 \& 1.0 \mathrm{~Hz}$ & $0.5 \& 1.5 \mathrm{~Hz}$ & $0.7 \& 1.1 \mathrm{~Hz}$ & $1.1 \& 1.7 \mathrm{~Hz}$ & $1.7 \& 2.1 \mathrm{~Hz}$ & Average \\
\hline \hline JSS, open & 2.0 & 1.4 & 3.4 & 3.0 & 2.0 & 3.6 & 4.0 & 5.0 & $\mathbf{3 . 1}$ \\
\hline VS, open & 1.4 & 1.2 & 3.0 & 1.2 & 1.8 & 2.6 & 3.2 & 5.0 & $\mathbf{2 . 4}$ \\
\hline JSS, closed & 1.8 & 1.4 & 3.4 & 2.4 & 2.8 & 3.4 & 4.6 & 5.0 & $\mathbf{3 . 1}$ \\
\hline VS, closed & 1.3 & 2.0 & 3.8 & 1.6 & 1.2 & 3.6 & 4.2 & 4.8 & $\mathbf{2 . 8}$ \\
\hline \hline Average & $\mathbf{1 . 6}$ & $\mathbf{1 . 5}$ & $\mathbf{3 . 4}$ & $\mathbf{2 . 1}$ & $\mathbf{2 . 0}$ & $\mathbf{3 . 3}$ & $\mathbf{4 . 0}$ & $\mathbf{5 . 0}$ & $\mathbf{2 . 8}$ \\
\hline
\end{tabular}

For consistent data analysis, all mirror motions (JSS) were flipped $(x=-x)$ so the positions would be directly comparable to the input. Also, the first two seconds were skipped since the input was not operating at full strength. Note that the time graphs in Fig. 2 show the entire time.

\section{B. Results}

The average performance ratings for the five subjects are shown in Table I. We conducted a one-way analysis of variance (ANOVA) to determine which symmetry mode and frequency subjects were able to accurately follow. When the ANOVA yielded significant results, we used Turkey's honest significance difference test for post hoc analyses. We used an alpha level of 0.05 for all statistical tests.
The effect of symmetry type and eye state was statistically significant, $F(3,4)=3.9, p=0.011$. The results are shown in Fig. 3. Post hoc analyses showed that VS eyes open performed significantly better than JSS, regardless of whether the eyes were open or closed. Within the VS mode, there was a large difference between eyes open and eyes closed, but the difference was not statistically significant. These results suggest that VS is easier to command and could be a beneficial mode of rehabilitation training, as examined in [27]. VS could possibly be more beneficial since the neural control operates closer to the visual task level [25], whereas JSS operates at the functional level. As opposed to working on controlling the joint motion directly, VS allows motions in the visual hand oriented space, which is where most activities of daily living are performed. 


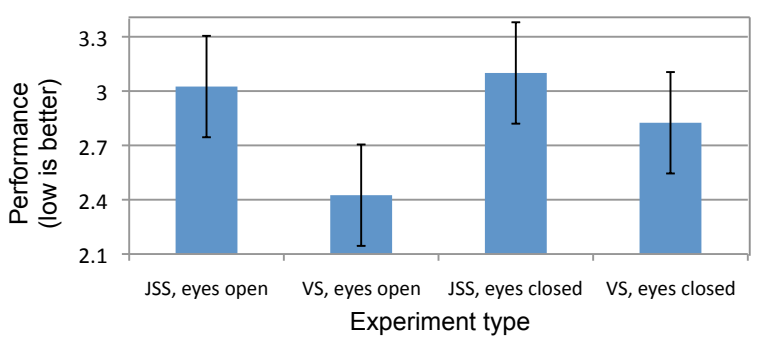

Fig. 3. The average performance for each experiment type is shown with $95 \%$ confidence intervals.

It is surprising that the JSS mode performed very similarly regardless of whether the eyes were open or closed. It makes sense that VS improved with eyes open since that mode operates in the visual space. However, JSS operates at the joint level where the motions are visually opposite, so we expected JSS eyes closed to have been better than JSS eyes open. In JSS, the individuals could have relied on proprioception more so than vision, but this does not appear to be the case.

As shown in Fig. 4, certain frequencies are too fast and/or complicated for subjects to recreate. There is a statistically significant difference between the input frequencies, $F(7,4)=32.8, p<0.001$. Post hoc analyses showed that subjects performed worse on the $1.7 \& 2.1 \mathrm{~Hz}$ superimposed frequencies than on the other seven frequencies. These fast superimposed non-harmonic frequencies are difficult to follow because the motion changes quickly and the relatively large repetition time makes the input appear more erratic.

There was no significant difference between the $2.0 \mathrm{~Hz}$, $0.7 \& 1.1 \mathrm{~Hz}$, and $1.1 \& 1.7 \mathrm{~Hz}$ frequencies. It is interesting to note that the $2.0 \mathrm{~Hz}$ trials and the $0.7 \& 1.1 \mathrm{~Hz}$ had a very similar performance rating. Similarly, the two harmonic, $0.5 \& 1.0 \mathrm{~Hz}$ and $0.5 \& 1.5 \mathrm{~Hz}$, and the two slowest, $0.5 \mathrm{~Hz}$ and $1.0 \mathrm{~Hz}$, frequencies were not significantly different and had the best overall performance rating. These four patterns were slow enough that the subjects were able to identify and accurately follow them. The performance similarities between the different types of patterns suggests that two superimposed slow frequencies can be as difficult as one faster frequency. We suspect that two slow frequencies could provide a better trajectory for rhythmic learning than one faster frequency since multiple frequencies would train multiple types of motion simultaneously, such as short and long reaching tasks, as opposed to one type of reaching task. However, this remains an open question.

The guiding force from the robot in this experiment was displayed to the subjects as a virtual spring $(200 \mathrm{~N} / \mathrm{m})$ connected to the desired trajectory. This spring force was not strong enough to impose the position upon the user's hand, so the subjects had to act like an admittance controller to interpret the force and move their other hand, which was not physically coupled, so as to follow the desired path. This brings up an unanswered question concerning the physical coupling between the human and robot as well as between the human's two hands. In previous bimanual

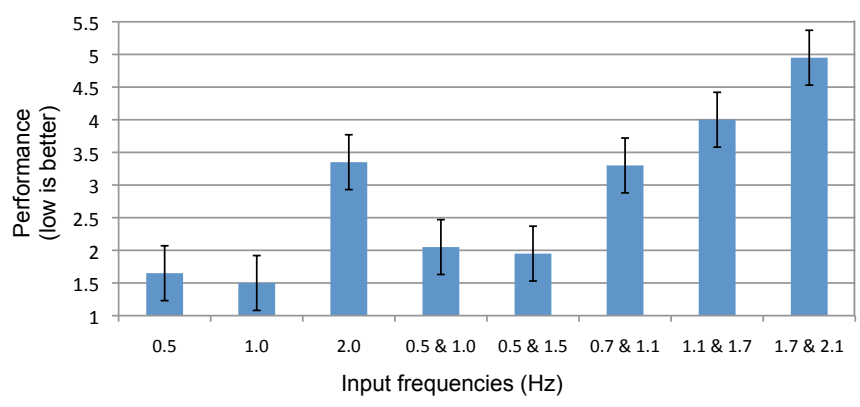

Fig. 4. The average performance for each input type is shown with $95 \%$ confidence intervals.

rehabilitation training methods, the connection between the hands has either been rigid [20], [27] or nonexistent [23]. The authors are not aware of any study comparing the effects of compliance in bimanual couplings for rehabilitation, but several studies have examined how compliance affects the perception of an object [28], [29]. If the physical coupling is completely rigid, the coupling would transmit all of the necessary force so the user would passively follow and could potentially not learn the path. However, if the coupling is too compliant, there would be no force transmitted and it would not be a physical coupling. To be effective for rehabilitation, the physical coupling between the hands needs to allow an appropriate amount of assistance force.

We acknowledge that there may be several factors affecting this study such as handedness and gender. In future studies with larger sample sizes, we will be able to report on these effects.

\section{COnClusions And Future Work}

To fully develop bimanual rehabilitation for home-use, several open questions remain concerning the physical coupling between the robot and the human's two arms. In this paper, we have begun to answer two of these questions. We compared two types of symmetric motions that could be used for bimanual rehabilitation: joint space (mirror) symmetry and visual symmetry. We demonstrated that visually symmetric motions were generally easier to perform, despite the biomechanical and neurological benefits associated with joint space symmetries, which suggests that visual symmetry may be a preferred method for rehabilitation. We also examined the type of rhythmic symmetries employed in rehabilitation and hypothesize that multiple slow harmonic frequencies could produce a more varied motor task that would retrain more motions for individuals following a stroke. The experiments performed here focused on two types of symmetries that exist in a one degree of freedom task. As we extend this work to include planar motions, we will also incorporate point mirror symmetries, which only exist in two or more degree of freedom tasks. We will also examine how the compliance of the physical coupling affects the performance.

The method presented in this paper is based on using one hand as a position input and the other hand as a position output. This technique can possibly be adapted for use in 
virtual trainers and simulators that recreate previous physical sensations. These environments suffer from a perceptual difference in the way individuals perceive a motion since being actively engaged in a task is different than being acted upon (i.e. active vs. passive sensing) [30]. Reaching for and touching an object is distinctly different than having an object touch you. This is particularly problematic when an expert wants to impart the feel of a task to a novice. Using both arms, it may be possible to transform a passive task into an active task by using one hand as an input receiving a reference trajectory while the other hand recreates this path and receives force feedback that was part of the original interaction. Although the individual will not be creating the original path, they will be actively generating the path and receiving feedback throughout that path in one arm, which should cause them to perceive the interaction more like an active task than a passive task.

\section{ACKNOWLEDGMENTS}

The authors thank Seok Hun Kim for his comments regarding this work.

\section{REFERENCES}

[1] C. P. Latimer, J. Keeling, B. Lin, M. Henderson, and L. A. Hale, "The impact of bilateral therapy on upper limb function after chronic stroke: a systematic review," Disability \& Rehabilitation, vol. 32, no. 15, pp. 1221-1231, 2010.

[2] L. Marchal-Crespo and D. Reinkensmeyer, "Review of control strategies for robotic movement training after neurologic injury," $J$ of NeuroEngineering and Rehabilitation, vol. 6, no. 1, p. 20, 2009.

[3] G. Kwakkel, B. Kollen, and H. Krebs, "Effects of Robot-Assisted Therapy on Upper Limb Recovery After Stroke: A Systematic Review," Neurorehabil Neural Repair, vol. 22, no. 2, pp. 111-121, 2008.

[4] V. Huang and J. Krakauer, "Robotic neurorehabilitation: a computational motor learning perspective," $J$ of NeuroEngineering and Rehabilitation, vol. 6, no. 1, p. 5, 2009.

[5] B. Bobath, Adult hemiplegia: Evaluation and treatment. London, UK: Heinemann Medical Books Ltd., 1970.

[6] M. Knott and D. Voss, Proprioceptive Neuromuscular Facilitation: Patterns and Techniques, 2ed, 2nd ed. New York, NY: Harper \& Row Publishers Inc., 1968.

[7] R. Oden, "Systematic therapeutic exercises in the management of the paralyses in hemiplegia," JAMA, vol. 23, pp. 828-833, 1918.

[8] S. L. Wolf, C. J. Winstein, J. P. Miller, E. Taub, G. Uswatte, D. Morris, C. Giuliani, K. E. Light, and D. Nichols-Larsen, "Effect of Constraint-Induced Movement Therapy on Upper Extremity Function 3 to 9 Months After Stroke: The EXCITE Randomized Clinical Trial," JAMA, vol. 296, no. 17, pp. 2095-2104, 2006.

[9] A. S. Merians, D. Jack, R. Boian, M. Tremaine, G. C. Burdea, S. V. Adamovich, M. Recce, and H. Poizner, "Virtual Reality-Augmented Rehabilitation for Patients Following Stroke," Phys Ther, vol. 82, no. 9, pp. 898-915, 2002.

[10] L. Legg and P. Langhorne, "Rehabilitation therapy services for stroke patients living at home: systematic review of randomised trials," The Lancet, vol. 363, pp. 352 - 356,, 2004.

[11] T. Ryan, P. Enderby, and A. S. Rigby, "A randomized controlled trial to evaluate intensity of community-based rehabilitation provision following stroke or hip fracture in old age," Clinical Rehabilitation, vol. 20, no. 2, pp. 123-131, 2006.
[12] S. Tyson and G. Turner, "Discharge and follow-up for people with stroke: what happens and why," Clinical Rehabilitation, vol. 14, no. 4, pp. 381-392, 2000.

[13] D. Reinkensmeyer, C. Pang, J. Nessler, and C. C. Painter, "Java therapy: Web-based robotic rehabilitation," Integration of Assistive Technology in the Information Age, vol. 9, pp. 66-71, 2001.

[14] M. Johnson, X. Feng, L. Johnson, and J. Winters, "Potential of a suite of robot/computer-assisted motivating systems for personalized, home-based, stroke rehabilitation," $J$ of NeuroEngineering and Rehabilitation, vol. 4, no. 1, p. 6, 2007.

[15] A. Timmermans, H. Seelen, R. Willmann, and H. Kingma, "Technology-assisted training of arm-hand skills in stroke: concepts on reacquisition of motor control and therapist guidelines for rehabilitation technology design," J of NeuroEngineering and Rehabilitation, vol. 6, no. $1,2009$.

[16] R. A. Schmidt and R. A. Bjork, "New conceptualizations of practice: Common principles in three paradigms suggest new concepts for training," Psychological Science, vol. 3, no. 4, pp. 207-217, 1992.

[17] J. L. Patton and F. A. Mussa-Ivaldi, "Robot-assisted adaptive training: Custom force fields for teaching movement patterns," IEEE Trans. Biomed. Eng, vol. 51, pp. 636-646, 2004.

[18] J. Liepert, I. Uhde, S. Grf, O. Leidner, and C. Weiller, "Motor cortex plasticity during forced-use therapy in stroke patients: a preliminary study," J of Neurology, vol. 248, pp. 315-321, 2001.

[19] G. F. Wittenberg, R. Chen, K. Ishii, K. O. Bushara, E. Taub, L. H. Gerber, M. Hallett, and L. G. Cohen, "Constraint-Induced Therapy in Stroke: Magnetic-Stimulation Motor Maps and Cerebral Activation,' Neurorehabil Neural Repair, vol. 17, no. 1, pp. 48-57, 2003.

[20] C. G. Burgar, P. S. Lum, P. C. Shor, and H. F. M. Van der Loos, "Development of robots for rehabilitation therapy: The palo alto va/stanford experience," J of Rehabilitation Research and Development, vol. 37, pp. 663-674, 2000.

[21] S. L. Wolf, D. E. LeCraw, and L. A. Barton, "Comparison of Motor Copy and Targeted Biofeedback Training Techniques for Restitution of Upper Extremity Function Among Patients with Neurologic Disorders," Physical Therapy, vol. 69, no. 9, pp. 719-735, 1989.

[22] S. Hesse, G. Schulte-Tigges, M. Konrad, A. Bardeleben, and C. Werner, "Robot-assisted arm trainer for the passive and active practice of bilateral forearm and wrist movements in hemiparetic subjects," Archives of Physical Medicine and Rehabilitation, vol. 84, no. 6, pp. $915-920,2003$.

[23] J. Whitall, S. M. Waller, K. H. C. Silver, and R. F. Macko, "Repetitive Bilateral Arm Training With Rhythmic Auditory Cueing Improves Motor Function in Chronic Hemiparetic Stroke," Stroke, vol. 31, no. 10, pp. 2390-2395, 2000.

[24] M. J. Johnson, H. F. M. Van der Loos, C. G. Burgar, P. Shor, and L. J. Leifer, "Experimental results using force-feedback cueing in robot-assisted stroke therapy," IEEE Trans on Neural Systems and Rehabilitation Eng, vol. 13, pp. 335-348, 2005.

[25] J. R. Flanagan and A. K. Rao, "Trajectory adaptation to a nonlinear visuomotor transformation: evidence of motion planning in visually perceived space," J Neurophysiol, vol. 74, no. 5, pp. 2174-2178, 1995.

[26] D. J. Reinkensmeyer, P. S. Lum, and S. L. Lehman, "Human control of a simple two-hand grasp," Biol. Cybern, vol. 67, no. 6, pp. 553-564, 1992.

[27] S. Hesse, C. Werner, M. Pohl, J. Mehrholz, U. Puzich, and H. I. Krebs, "Mechanical arm trainer for the treatment of the severely affected arm after a stroke," Am J Phys Med Rehabil, vol. 87, pp. 779-788, 2008.

[28] W. Song, M. Flanders, and J. F. Soechting, "Effect of compliance on haptic perception of curvature." Somatosensory \& Motor Research, vol. 21, no. 3/4, pp. 177 - 182, 2004.

[29] N. Gurari, K. J. Kuchenbecker, and A. M. Okamura, "Stiffness discrimination with visual and proprioceptive cues," in Proc. 3rd Joint Eurohaptics Conf. \& Symp. on Haptic Interfaces for Virtual Envir \& Teleoperator Systems (World Haptics), 2009, pp. 121-126.

[30] S. Shergill, P. Bays, C. Frith, and D. Wolpert, "Two eyes for an eye: The neuroscience of force escalation," Science, vol. 301, p. 187, 2003. 